\title{
Recurrent pregnancy loss: can factor V Leiden mutations be a cause
}

\author{
R R N Reddy, Deepti Mutreja, Nikhil Moorchung, Indrayani Mukhopadhyay \\ Department of Pathology and Gynecology, Command Hospital Air Force Bangalore, Bengaluru, India
}

The role of Factor $V$ Leiden (FVL) mutation in recurrent miscarriages has been disputed. It has been hypothesized that FVL mutation in patients with recurrent miscarriages is treatable. In this study, we evaluated 78 pregnant women for FVL mutations, among whom 50 had a history of recurrent miscarriages. Only 1 (2\%) of the woman was positive for heterozygous FVL mutation. The incidence of FVL mutations in patients with recurrent pregnancy loss had an odds ratio of 1.72 (95\% confidence interval, $0.0681-43.8257 ; P>0.05)$. However, the findings were not statistically significant. Thus, we suggest that FVL mutation study may not be included in the battery of tests for recurrent miscarriages in the Indian population.

Keywords: Factor V Leiden; Recurrent miscarriage

\section{Introduction}

Recurrent miscarriage or recurrent pregnancy loss (RPL) is defined by the American Society of Reproductive Medicine as 2 or more failed pregnancies [1]. RPL has several causes, including chromosomal abnormalities, uterine structural anomalies, chronic medical and surgical conditions, and endocrine disorders especially in the first trimester of pregnancy [2]. An extensive investigation may not provide a definite etiological cause of RPL in approximately $50-70 \%$ of cases [3]. The association of inherited and acquired thrombophilia with RPL is controversial.

Pregnancy is a physiological hypercoagulable state. The mechanism of this hypercoagulable state is not completely understood. The various theories proposed to explain this hypercoagulable state includes a hyper-estrogenic effect during pregnancy, hemodilution, and unknown placental factors [4]. When compounded with inherited or acquired thrombophil$\mathrm{ia}$, this hypercoagulable state may increase the risk of thrombosis, which leads to occlusion of the placental vasculature and fetal loss.

Heterozygous Factor $V$ Leiden (FVL) mutation is a common mutation that increases the relative risk of thrombosis by 1.8 to 2.6-fold in the general population [5]. The mechanism of increased risk of thrombosis is attributed to the activated protein C (APC) resistance of Factor $V$ protein due to mutations at specific sites of cleavage by APC. This leads to faulty anticoagulant mechanisms that shift the balance toward coagulation unchecked [6]. However, the association of the FVL mutation with RPL is controversial. An equal number of studies showed strong and weak associations with $\operatorname{RPL}[7,8]$. In addition, various studies have shown a low prevalence of FVL in the Asian population [9].

This study was conducted to evaluate FVL mutations in pregnant women with and without a history of RPL and estimate the relative risk of RPL in cases with FVL mutations.

\section{Materials and methods}

A total of 78 women were included in this point prevalence

Received: 2018.08.03. Revised: 2018.12.20. Accepted: 2019.02.14. Corresponding author: Nikhil Moorchung

Department of Pathology and Gynecology, Command Hospital Air Force Bangalore, Agram Post, Cambridge Layout, Bangalore, Karnataka 560007, India

E-mail: nikhilmoorchung@rediffmail.com

https://orcid.org/0000-0003-2381-7737

Articles published in Obstet Gynecol Sci are open-access, distributed under the terms of the Creative Commons Attribution Non-Commercial License (http://creativecommons. org/licenses/by-nc/3.0/) which permits unrestricted non-commercial use, distribution, and reproduction in any medium, provided the original work is properly cited.

Copyright $\odot 2019$ Korean Society of Obstetrics and Gynecology 


\section{Obstetrics \& Gynecology Science}

Vol. 62, No. 3, 2019

cross-sectional study. Informed consent was obtained from all the patients, and the study was approved by the institutional ethics committee. Pregnant women were then classified into 2 groups; groups A and B.

Group A included pregnant women with a history of 2 or more unexplained pregnancy losses in the first, second, or third trimester of pregnancy. Causes unrelated to thrombophilia were ruled out in these patients. Group B included pregnant women with 1 or more live births without a history of pregnancy loss and any other disease. Pregnancies occurring post treatment with Gonadotrophin-releasing hormone $(\mathrm{GnRH})$ analogues or any other ovulation-inducing drugs were excluded from both groups.

Testing for FVL mutations was performed using the polymerase chain reaction (PCR) restriction fragment length polymorphism technique as previously described [10]. Two $\mathrm{mL}$ of peripheral blood was collected in ethylenediaminetetraacetic acid. DNA was extracted from this peripheral blood and the PCR was performed. Wild-type amplicons yielded fragments of $241 \mathrm{bp}$; FVL heterozygotes resulted in fragments of 241, 209, and 32 bp; and FVL homozygotes resulted in fragments of 209 and $32 \mathrm{bp}$.

\section{Statistical analysis}

The sample size was calculated using the Epi Info software (version 7.2). By using an odds ratio of 5 at a $P$-value of 0.05 , the sample size was calculated as 53 . The odds ratio was based on previous studies that showed a strong association between the mutation and RPL.

Data collected in this study were analyzed using Microsoft Excel 2013, and the odds ratios was calculated to estimate the risk of association between FVL and RPL.

\section{Results}

Of the 106 pregnant women (56 cases and 50 controls), 78 had a FVL mutation, of whom 50 belonged to group A (RPL) and 28 belonged to group B (controls). The remaining patients were excluded because DNA testing could not be performed on their samples because either the samples for DNA extraction were not available or the quality of the template for the reaction was poor. The mean age of the patients with RPL was 26.8 years, and that of the control group was 27.6 years.

Of the 50 women with RPL, 18 were in the 6- to 11-week; 22 , in the 12- to 23-week; and 10, in the 24- to 37-week gestational age groups. In the control group, 13, 9, and 6 women were in the 6- to 11-week, 12- to 23-week, and 24to 37-week gestational age groups, respectively. Of the 50 women with RPL, 46 had a history of 2 miscarriages (89.2\%) and 4 had a history of 3 or more miscarriages (10.8\%).

Of the 50 women with RPL, 1 had a heterozygous FVL mutation. None of the women in the control group had a FVL mutation (Table 1).

\section{Discussion}

Most of the studies on FVL mutations and its association with RPL have been in Caucasian populations where the prevalence of the FVL heterozygous mutation is estimated to be approximately $11 \%$ [6]. Only few studies have analyzed the prevalence of FVL mutations in the Indian population and the associated risk of RPL. One study that included 40 cases reported a $10 \%$ prevalence of FVL mutation in cases of RPL [11]. However, the study was limited by the fact that a control group was not included in the study, making it difficult to estimate the risk of association. Another study reported a $4.76 \%$ prevalence of FVL mutation in cases of RPL [12]. The study was limited by the fact that it was conducted in an endogamous population.

The Indian population consists of several ethnic groups, and in this study, different populations were included, making it a heterogenous sample. In addition, in this study, both cases and controls were included. The prevalence of FVL mu-

Table 1. Prevalence of the FVL mutation

\begin{tabular}{lccc}
\hline Mutation type & $\begin{array}{c}\text { RPL (group A) } \\
\text { in \% }\end{array}$ & $\begin{array}{c}\text { Control (group B) } \\
\text { in \% }\end{array}$ & Odds ratio (95\% confidence interval) \\
\hline FVL heterozygotes & $2(50)$ & $0(28)$ & $1.72(0.0681-43.8257)$ \\
FVL homozygotes & $0(50)$ & $0(28)$ & - \\
\hline
\end{tabular}

FVL, Factor $V$ Leiden; RPL, recurrent pregnancy loss. 


\section{Obstetrics \& Gynecology Science}

R R N Reddy, et al. Factor V Leiden mutations in recurrent pregnancy loss

tation was $2 \%(1 / 50)$ in the RPL group and nil (0/28) in the control group. None of the women from either of the groups were positive for FVL homozygous mutations. The odds ratio for the risk of association of FVL heterozygous mutations with RPL was estimated to be 1.72 (95\% confidence interval, $0.0681-43.8257 ; P>0.05)$. The risk of association was not significant.

The lack of an association between FVL mutations and RPL has been described in other Indian studies [13-15]. The prevalence of the FVL mutation was $6.8 \%$ in the study group and $6.8 \%$ in the control group in a study involving 261 women with RPL and 345 controls. Similarly, the prevalence of the FVL mutation was $20 \%$ in the study group and $18.8 \%$ in the control group in a study involving 134 patients and 44 controls.

This relationship between the lack of FVL mutations and the incidence of RPL has emphasized the lack of an association between the 2 in the Indian population. Literature regarding the prevalence of the FVL mutation in the population states contradictory information, with some studies denying an association between FVL mutations and RPL [16-18] and other studies describing an association between the 2 [19]. However, testing for hereditary thrombophilia remains an important investigation in the workup of women with RPL. Protein $\mathrm{C}$ and $\mathrm{S}$ deficiencies remain important etiological factors in the pathogenesis of RPL $[19,20]$.

With the wide variation in the findings of different studies, it is likely that FVL mutations have no significant association with RPL. At present, we do not recommend inclusion of testing for FVL mutations in the battery of tests to evaluate RPL cases in Indian populations.

\section{Conflict of interest}

No potential conflict of interest relevant to this article was reported.

\section{Ethical approval}

Ethical Committee approval was received for this study from the Ethics Committee of Command Hospital Air Force Bangalore.

\section{Patient consent}

Informed consent was waived because of the retrospective study design.

\section{References}

1. Practice Committee of the American Society for Reproductive Medicine. Definitions of infertility and recurrent pregnancy loss. Fertil Steril 2008;89:1603.

2. Hatasaka HH. Recurrent miscarriage: epidemiologic factors, definitions, and incidence. Clin Obstet Gynecol 1994;37:625-34.

3. The American College of Obstetricians and Gynecologists. Frequently asked questions 100, pregnancy: repeated miscarriages [Internet]. Washington, D.C.: The American College of Obstetricians and Gynecologists; c2017 [cited 2017 Oct 25]. Available from: https://www. acog.org/ /media/For\%20Patients/faq100.pdf.

4. Brenner B. Haemostatic changes in pregnancy. Thromb Res 2004;114:409-14.

5. Mariar RA, Fink LM, Miller JL. Laboratory approach to thrombotic risk. In: McPherson RA, Pincus MR, editors. Henry's clinical diagnosis and management by laboratory methods. 22nd ed. New York (NY): Elsevier; 2011. p.825.

6. Kujovich JL. Factor $V$ Leiden thrombophilia. Genet Med 2011;13:1-16.

7. Mierla D, Szmal C, Neagos D, Cretu R, Stoian V, Jardan D. Association of prothrombin (A20210G) and Factor $V$ Leiden (A506G) with recurrent pregnancy loss. Maedica (Buchar) 2012;7:222-6.

8. Cardona H, Castañeda SA, Cardona Maya W, Alvarez L, Gómez J, Gómez JT, et al. Lack of association between recurrent pregnancy loss and inherited thrombophilia in a group of Colombian patients. Thrombosis 2012;2012:367823.

9. Herrmann FH, Koesling M, Schröder W, Altman R, Jimenez Bonilla R, Lopaciuk $S$, et al. Epidemiology of Factor $\mathrm{V}$ Leiden mutation in various populations: Northeastern Germany, Poland, Argentina, Venezuela, Costa Rica and India. In: Scharrer I, Schramm W, editors. 27. Hämophilie-Symposion Hamburg 1996. Berlin, Heidelberg: Springer; 1998. p.347-51.

10. Gandrille S, Alhenc-Gelas M, Aiach M. A rapid screening 


\section{Obstetrics \& Gynecology Science}

Vol. 62, No. 3, 2019

method for the factor $V$ Arg506-->Gln mutation. Blood Coagul Fibrinolysis 1995;6:245-8.

11. Kumar N, Ahluwalia J, Das R, Rohilla M, Bose S, Kishan $H$, et al. Inherited thrombophilia profile in patients with recurrent miscarriages: experience from a tertiary care center in North India. Obstet Gynecol Sci 2015;58:5147.

12. Mukhopadhyay R, Saraswathy KN, Ghosh PK. MTHFR C677T and Factor $V$ Leiden in recurrent pregnancy loss: a study among an endogamous group in North India. Genet Test Mol Biomarkers 2009;13:861-5.

13. Jaslow CR, Carney JL, Kutteh WH. Diagnostic factors identified in 1020 women with two versus three or more recurrent pregnancy losses. Fertil Steril 2010;93:123443.

14. Parand A, Zolghadri J, Nezam M, Afrasiabi A, Haghpanah S, Karimi M. Inherited thrombophilia and recurrent pregnancy loss. Iran Red Crescent Med J 2013;15:e13708.

15. Parveen F, Shukla A, Agrawal S. Should Factor V Leiden mutation and prothrombin gene polymorphism testing be done in women with recurrent miscarriage from
North India? Arch Gynecol Obstet 2013;287:375-81.

16. Pasquier E, Bohec C, Mottier D, Jaffuel S, Mercier B, Férec $C$, et al. Inherited thrombophilias and unexplained pregnancy loss: an incident case-control study. J Thromb Haemost 2009;7:306-11.

17. Altintas A, Pasa S, Akdeniz N, Cil T, Yurt M, Ayyildiz O, et al. Factor $V$ Leiden and G20210A prothrombin mutations in patients with recurrent pregnancy loss: data from the southeast of Turkey. Ann Hematol 2007;86:727-31.

18. Dilley A, Benito C, Hooper WC, Austin H, Miller C, ElJamil $M$, et al. Mutations in the Factor $V$, prothrombin and MTHFR genes are not risk factors for recurrent fetal loss. J Matern Fetal Neonatal Med 2002;11:176-82.

19. Pasińska M, Soszyńska K, Runge A, Dabrowska A, Juraszek $A$, Janiszewska $T$, et al. Molecular diagnostic tests for thrombophilia in patients referred to genetic counseling clinic because due to recurrent pregnancy failure. One center's experience. Ginekol Pol 2012;83:178-82.

20. Vora S, Shetty S, Salvi V, Satoskar P, Ghosh K. Thrombophilia and unexplained pregnancy loss in Indian patients. Natl Med J India 2008;21:116-9. 